\title{
Role of Platelets in Rheumatic Mitral Stenosis: An Observational Study
}

\author{
Shravan Kumar Chetti ${ }^{1} \quad$ Santhimayee Kalivela ${ }^{2} \quad$ Suresh Yerra ${ }^{1} \quad$ Indrani Garre ${ }^{1} \quad$ Achukatla Kumar $^{1}$ \\ ${ }^{1}$ Department of Cardiology, Nizam's Institute of Medical Sciences, \\ Punjagutta, Hyderabad, India \\ 2Department of Peadiatrics, Om Sai Hospital, Hyderabad, India

\begin{abstract}
Address for correspondence Shravan Kumar CH, DNB, DM, Cardiology Resident, Department of Cardiology, Nizam's Institute of Medical Sciences, Punjagutta 500082, Hyderabad, Telangana, India (e-mail: shravankch@gmail.com).
\end{abstract}

\begin{abstract}
Keywords

- mean platelet volume

- platelet distribution width

- mitral stenosis

- left atrial clot

Background As previous studies demonstrated, the mean platelet volume (MPV) is one of the determinant factors for the development of left atrial appendage (LAA) clot. The authors wanted to see other parameters such as platelet distribution width (PDW) along with MPV in patients with chronic rheumatic heart disease (CHRD) in whom transesophageal echocardiogram (TEE) was done.

Methods Total 50 consecutive CRHD patients with predominantly mitral stenosis (MS) came for cardiac outpatient department (OPD) for balloon valvuloplasty and were enrolled. Transthoracic echocardiography (TTE) and TEE were performed for each patient. Laboratory investigations included all biochemical parameters. In addition MPV and PDW were noted.

Results Most of the 50 patients enrolled in this study were middle-aged women $(n=37)$, with mean age of $40.18 \pm 9.50$ years. Patients were divided into two groups: with left atrial (LA) clot ( $n=24$, group 1$)$ and without LA clot ( $n=26$, group 2$)$. Out of them, male population with severe MS was more prone for LA clot formation $(p=0.031)$. When comparing severity of MS with electrocardiographic (ECG) rhythm (normal vs. atrial fibrillation), no statistically significant difference was observed with clot formation $(p=-0.44)$. In this study, presence of clot is more when the patient has larger-size platelets, that is, higher MPV, which is statistically significant $(9.823 \pm 0.759$ vs. $10.996 \pm 0.534 ; p=0.000)$. Severe MS is associated with high normal PDW whether the clot is present or not but statistically not significant $(p=-0.926)$.

Conclusion Severity of MS in CRHD patients is an independent determinant of LA clot formation irrespective of rhythm abnormalities and higher MPV value. Male patients with severe MS are more prone to LA clot formation.
\end{abstract}

\section{Introduction}

Mitral stenosis (MS) is the most common sequel of rheumatic fever that causes significant morbidity and mortality in developing countries. ${ }^{1}$ Systemic thromboembolism is one of the most dreaded complications of rheumatic MS. ${ }^{2}$ A hypercoagulable state is present in patients with rheumatic MS, and patients are prone to thromboembolic events despite treatment with oral anticoagulants. ${ }^{3}$ Platelets have an important role in the coagulation system, by activation of factors in intrinsic pathway, and also have a role in the hemostatic system. ${ }^{4,5}$ The mitral valve orifice area restriction leads to increased shear stress that is responsible for platelet activation in both the peripheral and left atrial (LA) blood of MS patients. ${ }^{6,7}$

Mean platelet volume (MPV) and platelet distribution width (PDW) are useful for predicting platelet function and activity. ${ }^{3,89}$ Large platelets have more aggregation and thromboxane $\mathrm{A}_{2}$ and thromboglobulin-releasing properties than small ones, which lead to high prothrombotic state. ${ }^{10-12}$ Elevated MPV level has been shown to be an independent risk factor for cardiovascular disease and thromboembolic events. MPV is easily measurable and acts as an index for 
platelet function. ${ }^{13}$ It has been shown that a higher MPV is correlated with a greater platelet activation. ${ }^{8}$

In a study done by Varol et al, ${ }^{14}$ MPV was significantly elevated in MS patients who were in sinus rhythm compared with control patients, which may be responsible for more thromboembolic events, but studies on PDW are still lacking.

The purpose of this study is to know MPV, PDW variation with severity of MS, and their relation with LA clot formation.

\section{Methods and Study Population}

Total 50 consecutive rheumatic MS patients who fulfilled following inclusion criteria after excluding from exclusion criteria were enrolled.

\section{Inclusion Criteria}

1. Patients age $>18$ years

2. Patients with chronic rheumatic heart disease (CHRD) who came to cardiac OPD

3. Patients excluding from exclusion criteria

\section{Exclusion Criteria}

1. Aortic and/or pulmonary valve disease

2. Left ventricular systolic dysfunction

3. Idiopathic thrombocytopenic purpura (ITP)

4. Chronic inflammatory conditions

5. Malignancy

6. Systemic or pulmonary thromboembolism

7. Congenital hemorrhagic disease

After enrolment of patients, detailed transthoracic echocardiography (TTE) at echo laboratory and later transesophageal echocardiography (TEE) with Philips IE 33 (Koninklijke Philips N.V.) with S5-1, S7-2 Omni probe, respectively, were performed according to American Society of Echocardiography (ASE) guidelines 2017.

\section{Criteria for Spontaneous Echo Contrast or Clot}

- Spontaneous echo contrast: Swirling echo density with the LA or left atrial appendage (LAA) imaged with gain set to diminish background
- Dense: Continuously seen

- Faint: Intermittent

- Sludge: Viscous, gelatinous morphology without consistent form

- Thrombus: Organized echo density with defined border, often oscillating

For all the patients enrolled in this study after TTE and TEE, blood samples were drawn and sent for biochemical analysis, erythrocyte sedimentation rate (ESR), complete blood picture, and platelet indices such as MPV and PDW. The measurements were performed immediately after blood sampling to prevent in vitro platelet activation. Informed consent was taken from all the study population.

\section{Statistical Analysis}

Data are demonstrated as mean \pm standard deviation (SD) for normally distributed continuous variables, median (minimum-maximum) for skew distributed continuous variables, and frequencies for categorical variables. Pearson chi-square test was performed for the comparison of categorical variables. A binary logistic regression analysis was done for multiple variables with clot formation. A $p$ value of $<0.05$ was con-sidered significant, and the confidence interval $(\mathrm{CI})$ was $95 \%$.

\section{Results}

Total 50 patients were enrolled in this study. Most of them were middle-aged women, with mean age of 40.18 \pm 9.50 (mini-mum of 19 years, maximum age 54). Initial blood parameters measured were with mean normal hemoglobin, leucocyte count, platelet count, and renal parameters (-Table $\mathbf{1}$ ).

Our study population had mean platelet count $2.090 \pm$ $0.4042 \mathrm{~L} / \mathrm{mm}^{3}$, mean MPV of $10.386 \pm 0.882 \mathrm{fL}$, and PDW of mean $16.686 \pm 0.588 \%$ (minimum 15.30 , maximum 18.00 with PDW median 16.700 (Q1: 16.200, Q3: 17.000) (-Table 2).

In this study population ( $n=50$ ), median platelet count of $200,000 / \mathrm{mm}^{3}$ (Q1: $187,500 / \mathrm{mm}^{3}$, Q3: $220,000 / \mathrm{mm}^{3}$ ) was noted (-Fig. 1).

Table 1 Variables in study population and their distribution

\begin{tabular}{|l|l|l|l|l|l|l|l|}
\hline Variable & Mean & SD & Minimum & Q1 & Median & Q3 & Maximum \\
\hline Age & 40.18 & 9.50 & 19.00 & 32.0 & 40.50 & 48.00 & 55.00 \\
\hline Hb & 12.332 & 1.421 & 9.600 & 11.0 & 12.100 & 13.62 & 15.700 \\
\hline PCV & 36.604 & 3.757 & 29.000 & 34.0 & 36.000 & 39.25 & 45.000 \\
\hline TLC & 8918 & 2401 & 5700 & 7400 & 8000 & 10100 & 17100 \\
\hline Platelet count & 2.090 & 0.4042 & 1.600 & 1.87 & 2.0000 & 2.200 & 3.4000 \\
\hline MPV & 10.386 & 0.882 & 8.6 & 9.6 & 10.700 & 11.02 & 11.800 \\
\hline Blood urea & 28.18 & 8.02 & 15 & 22.0 & 28.00 & 32 & 56.00 \\
\hline Serum creatinine & 0.890 & 0.2033 & 0.5 & 0.77 & 0.9000 & 1.00 & 1.500 \\
\hline ESR & 18.54 & 8.80 & 4.00 & 13.5 & 18.00 & 21.00 & 50.00 \\
\hline PDW & 16.686 & 0.588 & 16.200 & 16.2 & 16.700 & 17.00 & 18.000 \\
\hline
\end{tabular}

Abbreviations: ESR, erythrocyte sedimentation rate; Hb, hemoglobin; MPV, mean platelet volume; PCV, packed cell volume; PDW, platelet distribution width; SD, standard deviation; TLC, total leukocyte count. 
Table 2 Platelet variables in study population

\begin{tabular}{|l|l|l|l|l|l|l|l|}
\hline Variable & Mean & SD & Minimum & Q1 & Median & Q3 & Maximum \\
\hline Platelet count & 2.090 & 0.4042 & 1.600 & 1.87 & 2.0000 & 2.200 & 3.4000 \\
\hline MPV & 10.386 & 0.882 & 8.6 & 9.6 & 10.700 & 11.02 & 11.800 \\
\hline PDW & 16.686 & 0.588 & 16.200 & 16.2 & 16.700 & 17.00 & 18.000 \\
\hline
\end{tabular}

Abbreviations: MPV, mean platelet volume; PDW, platelet distribution width; SD, standard deviation.

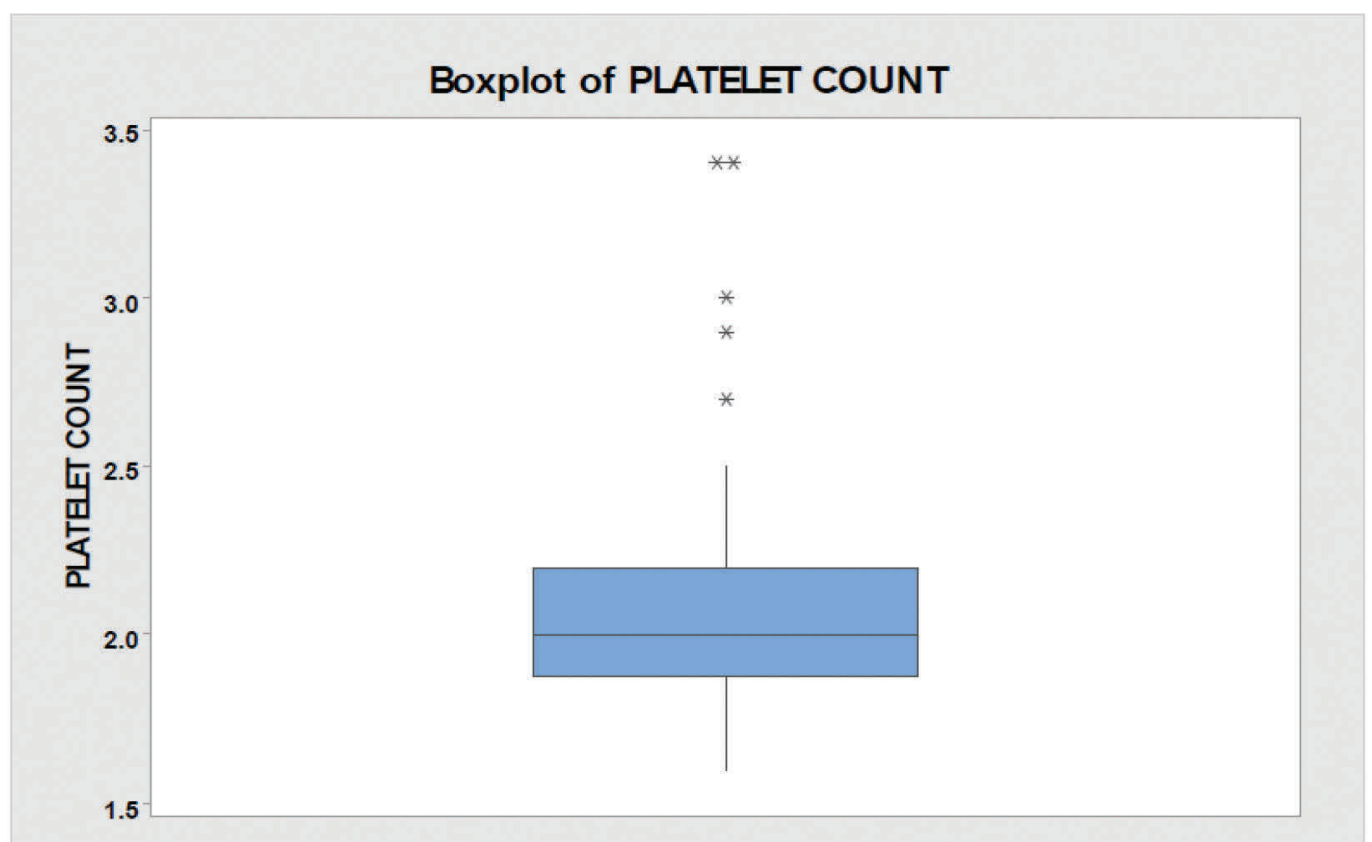

Fig. 1 Box plot of platelet count.

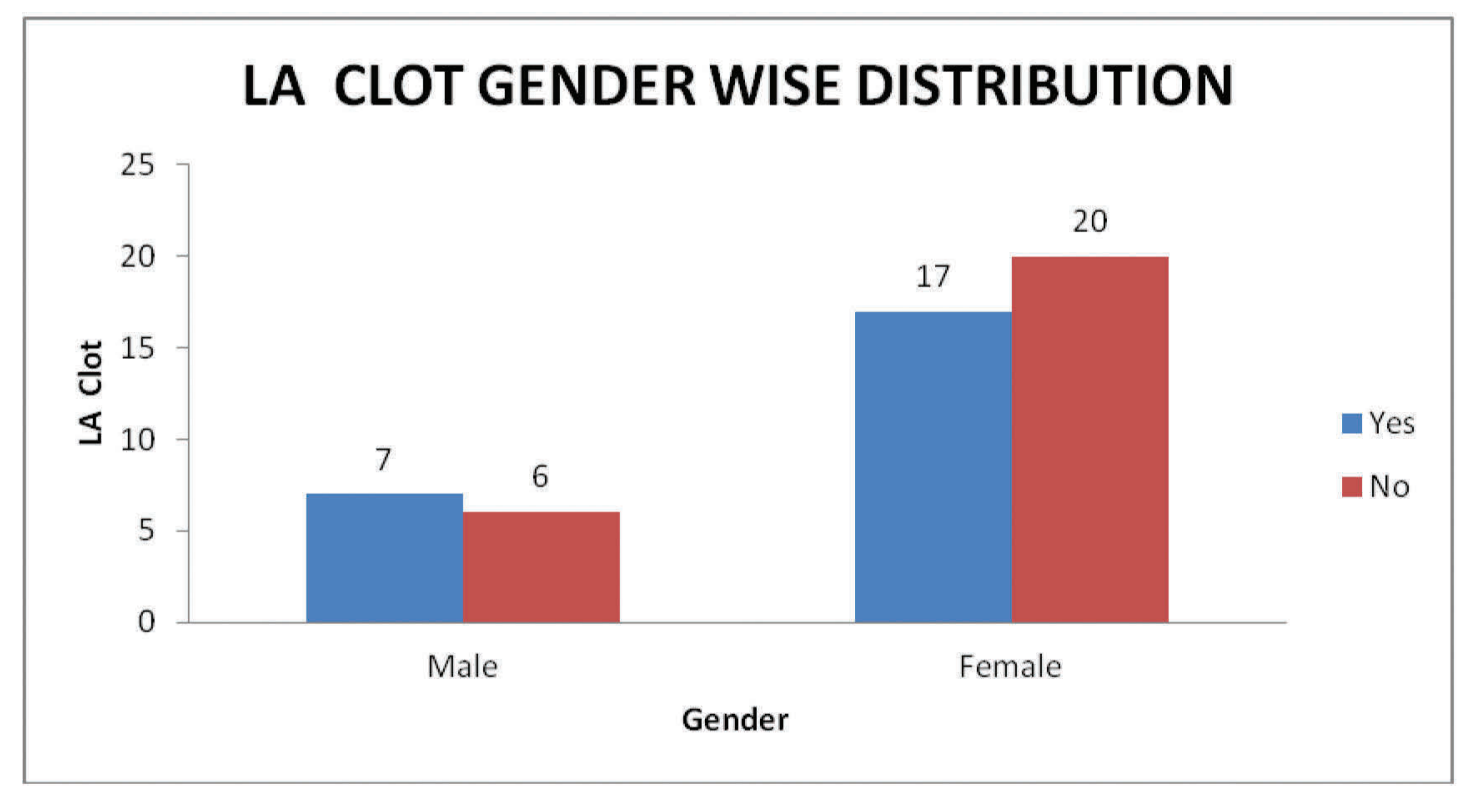

Fig. 2 Left atrial (LA) clot and gender-wise distribution.

In this study, it was observed that 6 out of 13 male patients and 20 out of 37 female patients had LA clot. Male patients with severe MS were more prone for LA clot formation, which was statistically significant $(p=0.031)$ ( - Fig. 2 ).
When comparing severity of MS mild $(n=1)$ versus severe $(n=23)$ with LA clot formation $(n=24)$, there was statistically significant effect observed with $p=0.000$ (95\% Cl for difference: $-0.792128,-0.388824)$ (-Table 3). 
Table 3 Severity of MS and LA clot

\begin{tabular}{|l|l|l|l|}
\hline $\begin{array}{l}\text { Severity of } \\
\text { MS }\end{array}$ & $\begin{array}{l}\text { LA clot } \\
\text { present }\end{array}$ & $\begin{array}{l}\text { LA clot } \\
\text { absent }\end{array}$ & Sample $p$ value \\
\hline Mild & 1 & 15 & 0.066667 \\
\hline Severe & 23 & 35 & 0.657143 \\
\hline
\end{tabular}

Abbreviations: LA, left atrial; MS, mitral stenosis.

When severity of MS with rhythm abnormalities was compared, no significant difference was observed with severity of MS and rhythm $(p=0.44)$ ( - Table 4$)$.

When comparing MPV with LA thrombus formation, presence of clot was greater when the patient had larger-size platelets, which was statistically significant (9.823 \pm 0.759 vs. $10.996 \pm 0.534 ; p=0.000)$ (-Fig. 3).

When comparing PDW with severity of MS, PDW normal range was divided into four quadrants. Of these, only 3 people were in third quadrant, that is, 13.95 to $15.92 \%$ of PDW and the remaining 47 MS cases were in the last quadrant, that is, 15.92 to $17.926 \%$ PDW ( - Fig. 4).

It means that severe MS is associated with normal level of the last quadrant PDW whether the clot is present or not but statistically not significant $(p-0.926)$.

When binary logistic regression analysis was done for other variables such as age, severity of mitral regurgitation (MR), hemoglobin ( $\mathrm{Hb})$, packed cell volume (PCV), total leukocyte count (TLC), platelet count, blood urea, and creatinine, no statistically significant difference was observed.
Table 4 Severity of MS with ECG rhythm

\begin{tabular}{|l|l|l|}
\hline ECG & Mild MS & Severe MS \\
\hline Normal sinus rhythm & 14 & 30 \\
\hline Atrial fibrillation & 1 & 5 \\
\hline
\end{tabular}

Abbreviations: ECG, electrocardiogram; MS, mitral stenosis.

When comparing severity of MS with electrocardiographic (ECG) rhythm (normal vs. atrial fibrillation), no statistically significant difference was observed with clot formation $(p-0.44)$ (-Table 5).

\section{Discussion}

Our study had predominantly middle-aged population that is expected in CHRD. Our population had normal renal parameters, hemoglobin, platelet counts, ESR, and leucocyte count. Most of them were enrolled after exclusion criteria and ruling out of infection as they are referred for balloon valvuloplasty/surgery. These results were consistent with study done by Akpek et al. ${ }^{9}$

Our study suggests that irrespective of rhythm abnormalities, severe MS itself is a determinant of LA clot formation, which is consistent with several studies that demonstrated that increased shear stress with turbulent flow in severe stenotic valves induce platelet activation. ${ }^{15,16}$ Our study results in consistent with study by Varol et al ${ }^{14}$ says that an increase in mitral valve area causes a decrease

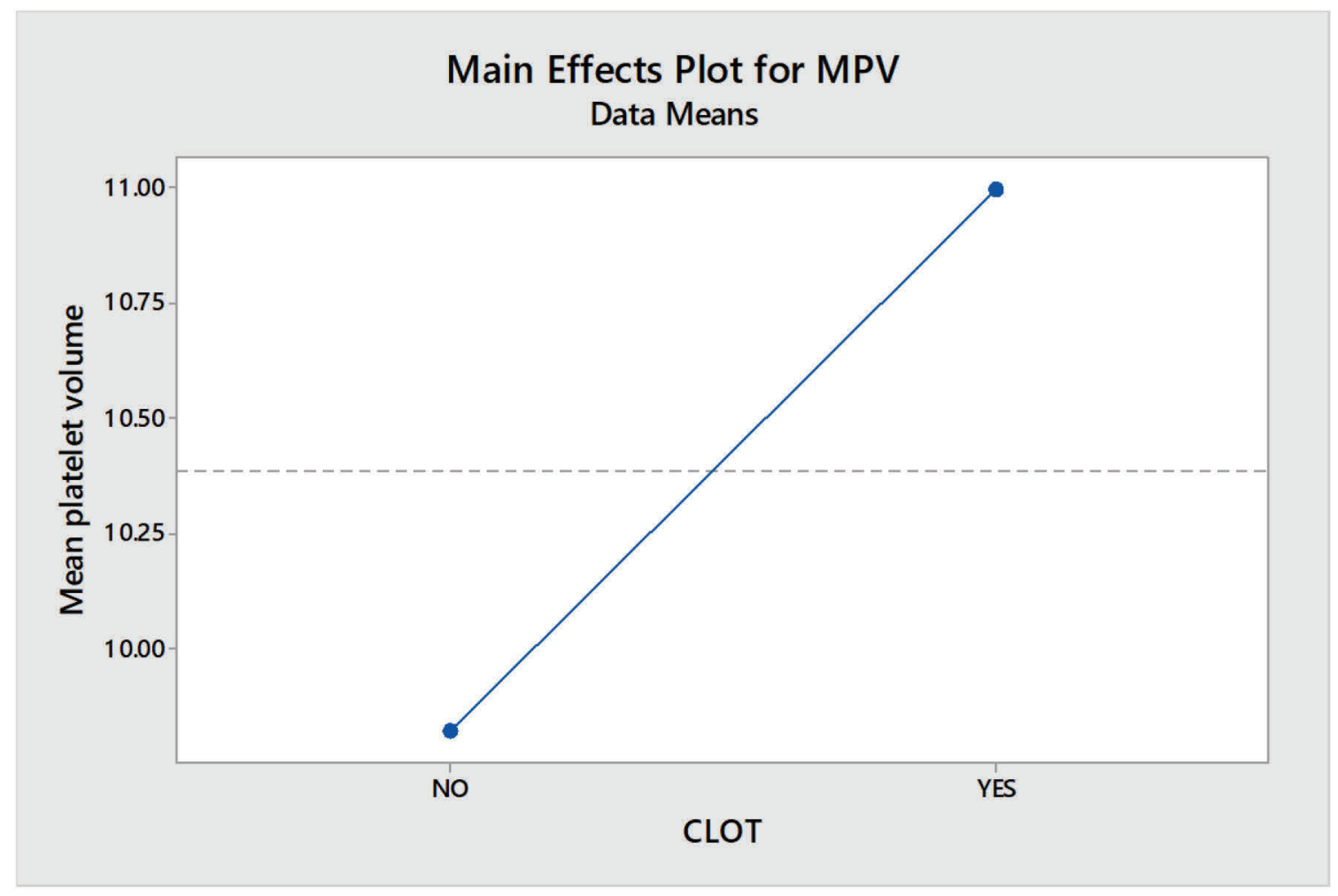

Fig. 3 Mean platelet volume (MPV) with effect on LA clot formation. 


\section{Boxplot of PDW}

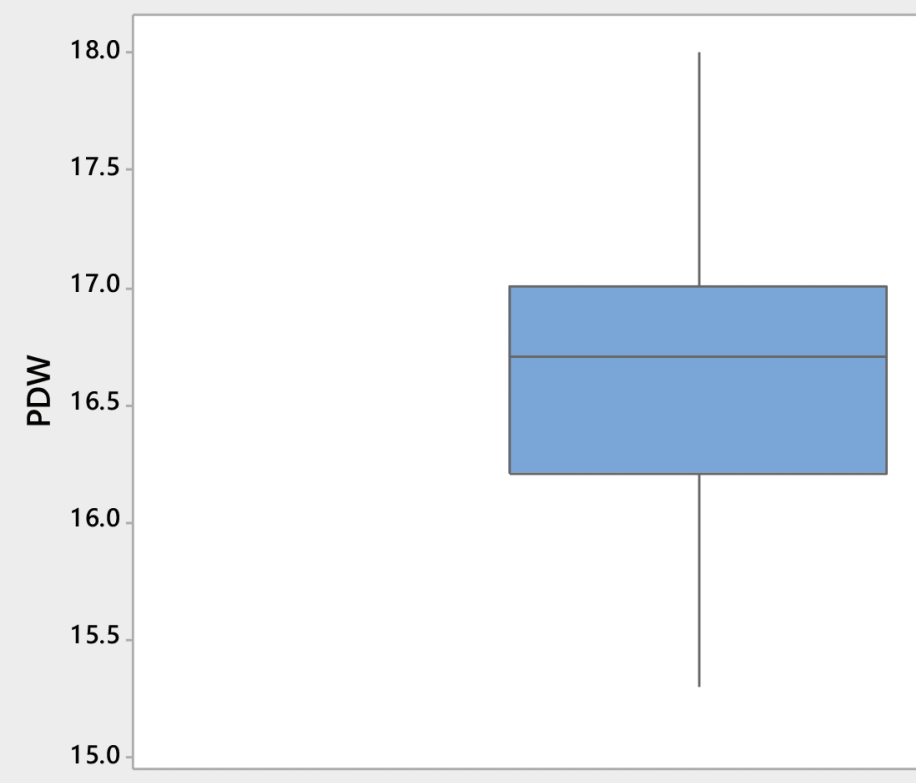

Fig. 4 Box plot of platelet distribution width (PDW).

Table 5 Binary logistic regression: clot versus PDW, Age, Hb, PCV, TLC, platelet count, MPV, and other parameters

\begin{tabular}{|l|l|l|}
\hline Source & Chi-square & $p$ Value \\
\hline Regression & 49.84 & 0.000 \\
\hline PDW & 0.01 & 0.926 \\
\hline Age & 1.49 & 0.222 \\
\hline Hb & 0.93 & 0.335 \\
\hline PCV & 0.74 & 0.391 \\
\hline TLC & 0.07 & 0.798 \\
\hline Platelet count & 0.86 & 0.354 \\
\hline MPV & 4.16 & 0.041 \\
\hline Blood urea & 0.29 & 0.592 \\
\hline Serum creatinine & 0.33 & 0.566 \\
\hline Severity of MS & 14.85 & 0.000 \\
\hline Sex & 4.65 & 0.031 \\
\hline Significance of MR & 0.13 & 0.723 \\
\hline
\end{tabular}

Abbreviations: Hb, hemoglobin; MPV, mean platelet volume; MR, mitral regurgitation; MS, mitral stenosis; PCV, packed cell volume; PDW, platelet distribution width; TLC, total leukocyte count.

in platelet activation and a decrease in mitral valve area causes an increase in platelet activation. Unlike other studies, our study did not find significant correlation between atrial fibrillation and LA clot, probably because of exclusion of patients with LA clot during TTE itself.

Our study suggests that most patients were middle aged, and that male patients with severe MS were more prone for clot formation, which is an incidental finding.

Our study suggests that patients with higher MPV are more prone for clot formation, and MPV as a independent predictor of LA clot formation, which is consistent with several studies that demonstrated that larger platelets that contain more prothrombotic materials (thromboxane $A_{2}$ and $B_{2}$ ) and have more glycoprotein IIb-IIIa receptor expression on the surface of platelets are more prone for aggregation. ${ }^{10-12}$

Jakubowski et al ${ }^{17}$ also reported that the presence of larger platelets decrease the inhibitory effectiveness of PGI2 on both platelet aggregation and release reaction, so larger platelets are more agreeable and metabolically and enzymatically more reactive than the smaller ones. It has also been shown that a higher MPV is correlated with a greater platelet activation, which is consistent with the study done by Yavuz et al. ${ }^{3}$

In our study, it was noted that PDW is in high normal range in all the study population, which suggests that severe MS is associated with high normal PDW whether the clot is present or not.

\section{Limitations of the Study}

As we included predominantly MS patients who came for further intervention, true estimation of prevalence cannot be made out. We included only patients in whom TTE was not suggestive of LA clot. We did not include patients with other rheumatic valvular diseases, and their impact was not known. As we excluded patients with LA clot during TTE itself, unlike other studies, correlation between LA clot and atrial fibrillation was not known in this study.

Left atrial appendage Doppler studies, velocities with platelet function were not performed in this study. We mainly focused on MPV and PDW parameters only, which is one of the major limitations of this study. 


\section{Conclusion}

Patients with severe MS are more prone for clot formation. Higher MPV value is an independent risk factor for clot formation and including routine screening of MPV and PDW may help in anticipation of LA clot formation and in management of patients.

Interpretation of study: From our study we suggest that patients with severe MS with higher MPV may benefit from antiplatelet therapy for prevention of thromboembolic events. Reducing MS severity by surgical or nonsurgical (balloon valvotomy) treatments may benefit from prevention of thromboembolism risk.

\section{Conflict of Interest}

None.

\section{References}

1 Takahashi T, Ono H, Warita K, et al. 10 years clinical outcomes in patients with mitral stenosis with unilateral commissural calcification treated with catheter balloon commissurotomy: single-center experience. J Cardiol 2008;51(1):33-41

2 Black IW, Hopkins AP, Lee LC, Walsh WF. Left atrial spontaneous echo contrast: a clinical and echocardiographic analysis. J Am Coll Cardiol 1991;18(2):398-404

3 Yavuz B, Ertuğrul DT, Yalçın AA, Küçükazman M, Ata N, Dal $\mathrm{K}$. Increased mean platelet volume in rheumatic mitral stenosis: a possible factor for thromboembolic events. J Cardiol 2009;53(2):204-207

4 Kunishima S, Hattori M, Kobayashi S, et al. Activation and destruction of platelets in patients with rheumatic heart disease. Eur Heart J 1994;15(3):335-338

5 Chen MC, Wu CJ, Yip HK, et al. Left atrial platelet activity with rheumatic mitral stenosis: correlation study of severity and platelet P-selectin expression by flow cytometry. Chest 2003;124(5):1663-1669
6 Yu SK, Latour JG, Marchandise B, Bois M. Shear stress-induced changes in platelet reactivity. Thromb Haemost 1979;40 (3):551-560

7 Peverill RE, Graham R, Gelman J, Yates LA, Harper RW, Smolich $\mathrm{JJ}$. Haematologic determinants of left atrial spontaneous echo contrast in mitral stenosis. Int J Cardiol 2001;81(2-3):235-242

8 Thompson CB, Jakubowski JA, Quinn PG, Deykin D, Valeri CR. Platelet size and age determine platelet function independently. Blood 1984;63(6):1372-1375

9 Akpek M, Kaya MG, Yarlioglues M, et al. Relationship between platelet indices and spontaneous echo contrast in patients with mitral stenosis. Eur J Echocardiogr 2011;12(11):865-870

10 Martin JF, Trowbridge EA, Salmon G, Plumb J. The biological significance of platelet volume: its relationship to bleeding time, platelet thromboxane B2 production and megakaryocyte nuclear DNA concentration. Thromb Res 1983;32(5):443-460

11 Jakubowski JA, Thompson CB, Vaillancourt R, Valeri CR, Deykin D. Arachidonic acid metabolism by platelets of differing size. Br J Haematol 1983;53(3):503-511

12 Giles H, Smith REA, Martin JF. Platelet glycoprotein IIb-IIIa and size are increased in acute myocardial infarction. Eur J Clin Invest 1994;24(1):69-72

13 Yarlioglues M, Kaya MG, Ardic I, et al. Relationship between mean platelet volume levels and subclinical target organ damage in newly diagnosed hypertensive patients. Blood Press 2011;20(2):92-97

14 Varol E, Ozaydin M, Turker Y, Alaca S. Mean platelet volume, an indicator of platelet activation, is increased in patients with mitral stenosis and sinus rhythm. Scand J Clin Lab Invest 2009;69(6):708-712

15 Smith RL, Blick EF, Coalson J, Stein PD. Thrombus production by turbulence. J Appl Physiol 1972;32(2):261-264

16 Stein PD, Sabbah HN. Measured turbulence and its effect on thrombus formation. Circ Res 1974;35(4):608-614

17 Jakubowski JA, Adler B, Thompson CB, Valeri CR, Deykin D. Influence of platelet volume on the ability of prostacyclin to inhibit platelet aggregation and the release reaction. J Lab Clin Med 1985;105(2):271-276 